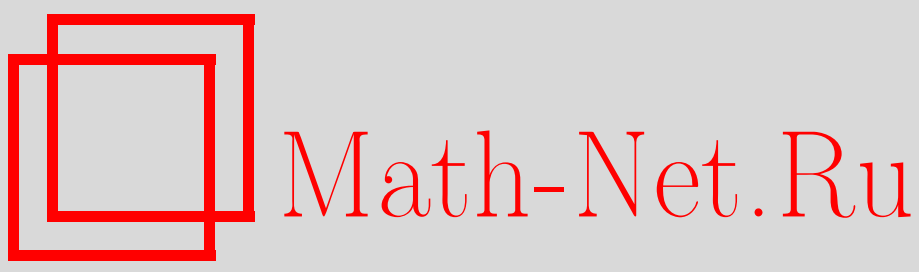

В. И. Лотов, Н. Г. Орлова, О числе пересечений полосы траекториями случайного блуждания, Матем. сб., 2003, том 194, номер 6, 135-146

DOI: https://doi.org/10.4213/sm746

Использование Общероссийского математического портала Math-Net.Ru подразумевает, что вы прочитали и согласны с пользовательским соглашением

http: //www. mathnet.ru/rus/agreement

Параметры загрузки:

IP : 107.22 .136 .117

26 апреля 2023 г., 18:35:28 
УДК 519.21

\author{
В.И. Лотов, Н.Г. Орлова
}

\title{
О числе пересечений полосы траекториями случайного блуждания
}

\begin{abstract}
Получены точные выражения для распределения общего числа пересечений полосы траекториями случайного блуждания, скачки которого имеют двустороннее геометрическое распределение. Распределение количества пересечений за конечньй промежуток времени найдено в явном виде для блужданий со скачками, принимающими значения \pm 1 . Доказана предельная теорема для совместного распределения числа пересечений расширяющейся полосы на конечном (pacтущем) промежу тке времени и положения блуждания в конце этого промежутка; найдено соответствующее предельное распределение.
\end{abstract}

Библиографиия: 8 названий.

\section{$\S 1$. Введение}

Пусть $\xi_{1}, \xi_{2}, \ldots$ - независимые одинаково распределенные случайные величины,

$$
S_{0}=0, \quad S_{n}=\xi_{1}+\cdots+\xi_{n} .
$$

В работе изучается число пересечений полосы $-a \leqslant y \leqslant b,-a \leqslant 0 \leqslant b$, на координатной плоскости точек $(x, y)$ траекторией случайного блуждания $\left\{\left(n, S_{n}\right)\right\}_{n=0}^{\infty}$. Известно, что число пересечений конечно с вероятностью единица, если сходится один из рядов

$$
\sum \frac{1}{n} \mathrm{P}\left(S_{n}>0\right)<\infty \quad \text { или } \quad \sum \frac{1}{n} \mathrm{P}\left(S_{n}<0\right)<\infty,
$$

для чего, в свою очередь, достаточно, чтобы выполнялось условие $\mathrm{E} \xi_{1} \neq 0$.

Пусть выполнено (1). Для рассматриваемого случайного блуждания определим моменты остановки (возможно, несобственные):

$$
\begin{gathered}
\tau_{0}^{+}=\tau_{0}^{-}=0, \quad \tau_{i}^{-}=\inf \left\{n \geqslant \tau_{i-1}^{+}: S_{n} \leqslant-a\right\}, \quad \tau_{i}^{+}=\inf \left\{n \geqslant \tau_{i}^{-}: S_{n} \geqslant b\right\} \\
i \geqslant 1 .
\end{gathered}
$$

Здесь всегда подразумевается, что inf $\varnothing=\infty$. Рассмотрим случайную величину $\eta^{(1)}$, равную числу пересечений указанной полосы снизу вверх траекторией $\left\{\left(n, S_{n}\right), n \geqslant 0\right\}$. Очевидно, $\mathrm{P}\left(\eta^{(1)} \geqslant k\right)=\mathrm{P}\left(\tau_{k}^{+}<\infty\right)$. Аналогичным образом

Работа вьполнена при финансовой поддержке Российского фонда фундаментальных исследований (грант № 02-01-00902) и фонда INTAS (грант № 00-265).

(C) В. И. Лотов, Н.Г. Орлова 2003 
определяется случайная величина $\eta^{(2)}$, равная числу пересечений полосы сверху вниз.

$\mathrm{B}$ [1] приведены представления вероятностей $\mathrm{P}\left(\eta^{(i)} \geqslant k\right)$ в терминах итераций некоторых операторов, связанных с компонентами факторизации функции $1-z \varphi(\lambda)$, $\varphi(\lambda)=\mathrm{E} \exp \left\{\lambda \xi_{1}\right\}$. Однако использование этих представлений затруднительно без дополнительных предположений о характере распределения $\xi_{1}$. В $\S 3$ настоящей работы найдены распределения случайных величин $\eta^{(i)}$ при условии, что $\xi_{i}$ целочисленны, $\mathrm{E} \xi_{1} \neq 0$, а вероятности $\mathrm{P}\left(\xi_{1}=k\right), \mathrm{P}\left(\xi_{1}=-k\right)$ убывают в геометрической прогрессии, что составляет содержание теоремы 1. Далее изучаются случайные величины $\eta_{n}^{(1)}, \eta_{n}^{(2)}$, также равные соответственно числу пересечений полосы снизу вверх и сверху вниз, но за промежуток времени от 0 до $n$. В теореме 2 для простейшего случайного блуждания получены точные формулы для распределений случайных величин $\eta_{n}^{(i)}, i=1,2$, без ограничений на среднее значение $\xi_{1}$.

Далее предполагается, что $\mathrm{E} \xi_{1}=0$. В этой ситуации обшее число пересечений полосы бесконечно с вероятностью единица. В условиях, когда случайная величина $\xi_{1}$ имеет произвольное распределение и конечный второй момент, доказана предельная теорема (теорема 3 ) о сходимости совместного распределения случайных величин $\eta_{n}^{(i)}$ и $S_{n}$ к соответствующим характеристикам винеровского процесса; последние найдены в явном виде. При этом предполагается, что шшрина полосы и длина рассматриваемого промежутка времени неограниченно возрастают согласованным образом.

Некоторые предельные результаты для числа пересечений полосы в случае нулевой ширины (полоса вырождается в прямую) изложены в работах [2], [3]. Работа [4] содержит утверждение о предельном распределении числа нулей в последовательности $S_{n}$ в случае, когда $\mathrm{P}\left(\xi_{1}=1\right)=\mathrm{P}\left(\xi_{1}=-1\right)=1 / 2 . \mathrm{B}[1]$ кроме упомянутых операторных представлений для вероятностей $\mathrm{P}\left(\eta^{(i)} \geqslant k\right)$ найдены их асимптотические представления при условии, что полоса безгранично расширяется. При этом дополнительно предполагалось выполненным условие Крамера на распределение скачков рассматриваемого блуждания.

\section{§ 2. Предварительные сведения}

В [1] установлено, что при $|z|<1, \operatorname{Re} \lambda=0$

$$
\mathrm{E}\left(z^{\tau_{k}^{+}} e^{\lambda S} \tau_{k}^{+} ; \tau_{k}^{+}<\infty\right)=\left(\left(J_{b}^{+} J_{-a}^{-}\right)^{k} e\right)(z, \lambda)
$$

где $e(\lambda) \equiv 1$, а операторы $J_{b}^{+}, J_{-a}^{-}$определяются следуюшим образом.

Пусть $1-z \varphi(\lambda)=r_{z+}(\lambda) \cdot r_{z-}(\lambda)$ - разложение на положительную и отрицательную компоненты факторизации при $|z|<1, \operatorname{Re} \lambda=0$ (см. например, [5]), где функция $r_{z+}(\lambda)$ отлична от нуля и аналитична при $\operatorname{Re} \lambda<0$, непрерьвна на множестве $\operatorname{Re} \lambda \leqslant 0$. Аналогичными свойствами в правой полуплоскости обладает $r_{z-}(\lambda)$, и такая факторизация единственна, если потребовать, например, $r_{z-}(+\infty)=1$. Пусть на прямой $\operatorname{Re} \lambda=0$ функция $g(\lambda)$ допускает представление

$$
g(\lambda)=\int_{-\infty}^{\infty} e^{\lambda y} d G(y)
$$


в котором функция $G$ имеет конечную полную вариацию, тогда при $\operatorname{Re} \lambda=0$ полагаем

$$
\begin{aligned}
& \left(J_{t}^{-} g\right)(z, \lambda)=r_{z-}(\lambda)\left[r_{z-}^{-1}(\lambda) g(\lambda)\right]^{(-\infty, t]}, \\
& \left(J_{t}^{+} g\right)(z, \lambda)=r_{z+}(\lambda)\left[r_{z+}^{-1}(\lambda) g(\lambda)\right]^{[t, \infty)} .
\end{aligned}
$$

Здесь используются обозначения вида

$$
\left[\int_{-\infty}^{\infty} e^{\lambda y} d G(y)\right]^{D}=\int_{D} e^{\lambda y} d G(y), \quad D \subset \mathbb{R}
$$

Функция $g$ может зависеть от $z$. Для краткости зависимость от $z$ в обозначениях операторов $J_{t}^{+}, J_{t}^{-}$не подчеркивается. Ясно, что для нахождения $\mathrm{P}\left(\tau_{k}^{+}<\infty\right)$ достаточно положить $\lambda=0$ в (2) и устремить $z$ к единице. Аналогичным образом находится вероятность события $\left\{\tau_{k}^{-}<\infty\right\}$.

\section{§3. Основные результаты}

Заметим вначале, что вероятности $\mathrm{P}\left(\eta^{(i)} \geqslant k\right)$ всегда могут быть оценены сверху соответствующими вероятностями для геометрического распределения. Пусть, к примеру, $\mathrm{E} \xi_{1}<0$. Тогда

$$
\mathrm{P}\left(\eta^{(1)} \geqslant k\right) \leqslant[\mathrm{P}(S \geqslant a+b)]^{k},
$$

где $S=\sup _{n \geqslant 0} S_{n}$. Действительно, обозначив $\nu_{t}=\inf \left\{n \geqslant 1: S_{n} \leqslant t\right\}$, будем иметь

$$
\begin{aligned}
\mathrm{P}\left(\eta^{(1)} \geqslant k\right) & =\int_{b}^{\infty} \mathrm{P}\left(S_{\tau_{k-1}^{+}} \in d y\right) \int_{-\infty}^{-a-y} \mathrm{P}(S \geqslant b-x) \mathrm{P}\left(S_{\nu_{-a-y}} \in d x\right) \\
& \leqslant \mathrm{P}(S \geqslant a+b) \int_{b}^{\infty} \mathrm{P}\left(S_{\nu_{-a-y}} \leqslant-a-y\right) \mathrm{P}\left(S_{\tau_{k-1}^{+}} \in d y\right) \\
& =\mathrm{P}(S \geqslant a+b) \mathrm{P}\left(\eta^{(1)} \geqslant k-1\right) \leqslant[\mathrm{P}(S \geqslant a+b)]^{k} .
\end{aligned}
$$

При $\mathrm{E} \xi_{1}>0$ по той же схеме получаем

$$
\mathrm{P}\left(\eta^{(1)} \geqslant k\right) \leqslant\left[\mathrm{P}\left(\inf _{n \geqslant 0} S_{n} \leqslant-a-b\right)\right]^{k} .
$$

Оценки для $\mathrm{P}\left(\eta^{(2)} \geqslant k\right)$ получаются аналогично.

Следующая теорема показывает, что случайные величины $\eta^{(i)}$ имеют в точности геометрическое распределение, если такой же характер распределения на каждой из полуосей имеют скачки блуждания $\xi_{i}$.

ТЕОРема 1. Пусть $a, b$ - натуральные числа и при $k \geqslant 1$

$$
\begin{gathered}
\mathrm{P}\left(\xi_{1}=k\right)=\alpha p^{k-1}, \quad \mathrm{P}\left(\xi_{1}=-k\right)=\beta q^{k-1}, \quad \mathrm{P}\left(\xi_{1}=0\right)=r, \\
\frac{\alpha}{1-p}+\frac{\beta}{1-q}=1-r .
\end{gathered}
$$


Здесь $p \geqslant 0, q \geqslant 0, r \geqslant 0, \alpha>0, \beta>0$. Предположим, ито $\mathrm{E} \xi_{1} \neq 0$. Тогда для любого $k \geqslant 1$

$$
\mathrm{P}\left(\eta^{(i)} \geqslant k\right)=c_{i} d^{k-1}
$$

əдe

$$
\begin{aligned}
c_{1} & =h_{1}^{a-1} \frac{h_{1}-q}{q-h_{2}} \frac{p h_{2}-1}{h_{2}^{a+b-1}} \frac{1}{1-p}, \\
c_{2} & =\left(1-p h_{2}\right) \frac{1}{h_{2}^{b}} h_{1}^{b+a-1} \frac{h_{1}-q}{1-p h_{1}} \frac{1}{1-q}, \\
d & =\frac{h_{1}-q}{q-h_{2}} \frac{p h_{2}-1}{1-p h_{1}}\left(\frac{h_{1}}{h_{2}}\right)^{b+a-1},
\end{aligned}
$$

$h_{1}, h_{2}$ - корни уравнения $\mathrm{E} \mu^{\xi_{1}}=1, h_{1}<h_{2}$ (одно из этих чисел всегда равно единиче).

ДокАЗАТЕльСтво. Нам будет удобнее перейти в (2) к переменной $\mu=e^{\lambda}$. При этом мы сохраним прежние обозначения для компонент факторизации и операторов $J_{t}^{ \pm}$как функций переменной $\mu$. Обозначим $\psi(\mu)=\mathrm{E} \mu^{\xi_{1}}$, тогда

$$
\begin{aligned}
1-z \psi(\mu) & =1-z\left[r+\frac{\alpha \mu}{1-\mu p}+\frac{\beta}{\mu-q}\right] \\
& =\frac{(1-\mu p)(\mu-q)-z r(1-\mu p)(\mu-q)-z \alpha \mu(\mu-q)-z \beta(1-\mu p)}{(1-\mu p)(\mu-q)} \\
& =\frac{\mu^{2} C(z)+\mu L(z)+K(z)}{(1-\mu p)(\mu-q)},
\end{aligned}
$$

где

$$
\begin{aligned}
C(z) & =-p+r z p-\alpha z, \\
L(z) & =1+p q+z(-r-r p q+\alpha q+\beta p), \\
K(z) & =-q+z(r q-\beta) .
\end{aligned}
$$

Заметим, что множитель при $\mu^{2}$ не может быть тождественным нулем, поскольку $C(z)<0$ при $0 \leqslant z \leqslant 1$. Таким образом,

$$
1-z \psi(\mu)=\frac{\mu-h_{1}(z)}{\mu-q} \frac{\mu-h_{2}(z)}{(1-\mu p) \widetilde{C}(z)}=r_{z-}(\mu) \cdot r_{z+}(\mu) .
$$

Здесь $h_{1,2}(z)$ - корни уравнения $1-z \psi(\mu)=0, q<h_{1}(z)<1<h_{2}(z)<1 / p$ при $z \in(0,1), C(z)=\widetilde{C}^{-1}(z)$. Приведенная факторизация обладает всеми указанными выше свойствами. Далее нам потребуются две леммы (ср. с леммами 2,3 в [6]).

ЛЕмма 1. Пусть ряд $g(\mu)=\sum_{-\infty}^{0} a_{k} \mu^{k}$ абсолютно сходится при $|\mu| \geqslant 1$. Тогда при $|z|<1,|\mu|=1$

$$
\left(J_{b}^{+} g\right)(z, \mu)=\frac{1-p h_{2}(z)}{h_{2}^{b}(z)} \frac{\mu^{b}}{1-\mu p} g\left(h_{2}(z)\right) .
$$


ДоКАЗАТЕЛЬСТво. Прямыми вычислениями устанавливаем:

$$
\begin{aligned}
{\left[_{z+}^{-1}(\mu) g(\mu)\right]^{[b, \infty)} } & =\widetilde{C}(z)\left[\frac{1-\mu p}{\mu-h_{2}(z)} \sum_{k=-\infty}^{0} a_{k} \mu^{k}\right]^{[b, \infty)} \\
& =\widetilde{C}(z)\left[\left(-p+\frac{1-p h_{2}(z)}{\mu-h_{2}(z)}\right) \sum_{k=-\infty}^{0} a_{k} \mu^{k}\right]^{[b, \infty)} \\
& =\widetilde{C}(z)\left[\frac{1-p h_{2}(z)}{-h_{2}(z)\left(1-\mu / h_{2}(z)\right)} \sum_{k=-\infty}^{0} a_{k} \mu^{k}\right]^{[b, \infty)} \\
& =\widetilde{C}(z) \frac{1-p h_{2}(z)}{-h_{2}(z)}\left[\sum_{i=0}^{\infty}\left(\frac{\mu}{h_{2}(z)}\right)^{i} \sum_{k=-\infty}^{0} a_{k} \mu^{k}\right]^{[b, \infty)} \\
& =\widetilde{C}(z) \frac{1-p h_{2}(z)}{-h_{2}(z)} \sum_{i=-\infty}^{0} a_{i} h_{2}^{i}(z) \sum_{k=b}^{\infty}\left(\frac{\mu}{h_{2}(z)}\right)^{k} \\
& =\widetilde{C}(z) \frac{1-p h_{2}(z)}{\mu-h_{2}(z)} g\left(h_{2}(z)\right) \frac{\mu^{b}}{h_{2}^{b}(z)}, \\
r_{z+}(\mu)\left[r_{z+}^{-1}(\mu) g(\mu)\right]^{[b, \infty)} & =\frac{\mu-h_{2}(z)}{(1-\mu p) \widetilde{C}(z)} \widetilde{C}(z) \frac{1-p h_{2}(z)}{\mu-h_{2}(z)} g\left(h_{2}(z)\right) \frac{\mu^{b}}{h_{2}^{b}(z)} \\
& =\frac{1-p h_{2}(z)}{1-\mu p} g\left(h_{2}(z)\right) \frac{\mu^{b}}{h_{2}^{b}(z)} .
\end{aligned}
$$

Лемма 1 доказана.

Лемма 2. Пусть ряд $g(\mu)=\sum_{0}^{\infty} a_{k} \mu^{k}$ абсолютно сходится при $|\mu| \leqslant 1$. Тогда при $|z|<1,|\mu|=1$

$$
\left(J_{-a}^{-} g\right)(z, \mu)=\frac{h_{1}(z)-q}{\mu-q}\left(\frac{h_{1}(z)}{\mu}\right)^{a-1} g\left(h_{1}(z)\right) .
$$

Лемма 2 доказывается аналогично.

Обозначим

$$
g_{1}(\mu)=\frac{\mu^{b}}{1-\mu p}, \quad g_{2}(\mu)=\frac{1}{\mu^{a-1}(\mu-q)} .
$$

Применяя леммы 1 и 2 к функциям $e(\mu), g_{1}(\mu), g_{2}(\mu)$, будем иметь

$$
\begin{aligned}
\left(J_{b}^{+} e\right)(z, \mu) & =\frac{1-p h_{2}(z)}{h_{2}^{b}(z)} g_{1}(\mu) \\
\left(J_{b}^{+} g_{2}\right)(z, \mu) & =\frac{p h_{2}(z)-1}{q-h_{2}(z)} \frac{1}{h_{2}^{a+b-1}(z)} g_{1}(\mu) \\
\left(J_{-a}^{-} e\right)(z, \mu) & =h_{1}^{a-1}(z)\left(h_{1}(z)-q\right) g_{2}(z) \\
\left(J_{-a}^{-} g_{1}\right)(z, \mu) & =\frac{h_{1}(z)-q}{1-p h_{1}(z)} h_{1}^{a+b-1}(z) g_{2}(\mu)
\end{aligned}
$$


и, следовательно, для любого $k \geqslant 1$

$$
\begin{aligned}
\left(\left(J_{b}^{+} J_{-a}^{-}\right)^{k} e\right)(z, \mu)= & h_{1}^{a-1}(z)\left(h_{1}(z)-q\right) \frac{p h_{2}(z)-1}{q-h_{2}(z)} \frac{1}{h_{2}^{a+b-1}(z)} \\
& \times\left(\frac{p h_{2}(z)-1}{q-h_{2}(z)}\left(\frac{h_{1}(z)}{h_{2}(z)}\right)^{a+b-1} \frac{h_{1}(z)-q}{1-p h_{1}(z)}\right)^{k-1} g_{1}(\mu), \\
\left(\left(J_{-a}^{-} J_{b}^{+}\right)^{k} e\right)(z, \mu)= & \left(1-p h_{2}(z)\right) \frac{h_{1}^{a+b-1}(z)}{h_{2}^{b}(z)} \frac{h_{1}(z)-q}{1-p h_{1}(z)} \\
& \times\left(\frac{p h_{2}(z)-1}{q-h_{2}(z)}\left(\frac{h_{1}(z)}{h_{2}(z)}\right)^{a+b-1} \frac{h_{1}(z)-q}{1-p h_{1}(z)}\right)^{k-1} g_{2}(\mu) .
\end{aligned}
$$

Отсюда получаем

$$
\begin{aligned}
& \mathrm{P}\left(\eta^{(1)} \geqslant k\right)=\lim _{z \rightarrow 1}\left(\left(J_{b}^{+} J_{-a}^{-}\right)^{k} e\right)(z, 1)=c_{1} d^{k-1} \\
& \mathrm{P}\left(\eta^{(2)} \geqslant k\right)=\lim _{z \rightarrow 1}\left(\left(J_{-a}^{-} J_{b}^{+}\right)^{k} e\right)(z, 1)=c_{2} d^{k-1} .
\end{aligned}
$$

Постоянные $c_{1}, c_{2}, d$ находятся из $(3),(4)$ при $\mu=1, z \rightarrow 1$. А именно $h_{1}=h_{1}(1)$, $h_{2}=h_{2}(1)$,

$$
\begin{gathered}
c_{1}=h_{1}^{a-1} \frac{h_{1}-q}{q-h_{2}} \frac{p h_{2}-1}{h_{2}^{a+b-1}} \frac{1}{1-p}, \quad c_{2}=\left(1-p h_{2}\right) \frac{1}{h_{2}^{b}} h_{1}^{a+b-1} \frac{h_{1}-q}{1-p h_{1}} \frac{1}{1-q} \\
d=\frac{h_{1}-q}{q-h_{2}} \frac{p h_{2}-1}{1-p h_{1}}\left(\frac{h_{1}}{h_{2}}\right)^{a+b-1} .
\end{gathered}
$$

Теорема 1 доказана.

ЗАмечАния. 1. Доказательство теоремы с очевидными изменениями может быть проведено для случая, когда $\mathrm{P}\left(\xi_{i} \geqslant x\right)=\alpha e^{-p x}, \mathrm{P}\left(\xi_{i}<x\right)=\beta e^{-q x}, x \geqslant 0$, и также приведет к геометрическому распределению для $\eta^{(i)}$.

2. Доказательство теоремы основьвается на возможности вычисления в явном виде функций вида $\left(J_{t}^{ \pm} g\right)(z, \mu)$ с последующим анализом суперпозиций операторов $J_{t}^{ \pm}$. Для этих целей предполагалось, что компоненты факторизации являются дробно-линейными функциями. Все вычисления могут быть проделаны и в более общей ситуации, когда функция $\psi(\mu)$ (или $\varphi(\lambda))$ рациональна (например, когда $\xi_{1}-$ ограниченная целочисленная величина). При этом компоненты факторизации также будут рациональными функциями и, следовательно, допускают разложение на простые дроби. Это также дает возможность вычислять в явном виде значения операторов $J_{t}^{ \pm}$и их суперпозиций, однако результат получится слишком громоздким, и по этой причине мы его не приводим. Отметим, что распределение числа пересечений полосы в этом случае будет являть собой смесь дискретных аналогов гамма-плотностей.

3. Требование $0 \in[-a, b]$ не принципиально. Формулы типа (5), (6) легко получаются и в случае, когда $0 \notin[-a, b]$. Замечания по этому поводу содержатся в [1]. 
Ясно, что общее число пересечений полосы будет неограниченно возрастать, если устремить к нулю снос случайного блуждания. Известно достаточно большое число публикаций, в которых изучается предельное поведение различных функционалов от траекторий случайного блуждания в этой ситуации. Полученные на этом пути результаты часто используются для описания функционирования систем обслуживания в условиях большой нагрузки. Мы воспользуемся наличием точных формул для распределения числа пересечений полосы, чтобы также получить предельный результат при $\mathrm{E} \xi_{1} \rightarrow 0$.

СлЕДСТВИЕ 1. Пусть выполнены условия теоремы 1 и $1-r \geqslant \varepsilon>0$. Обозначим $\theta=h_{2}-h_{1}$. Тогда для любого $t \geqslant 0, i=1,2$,

$$
\mathrm{P}\left(\theta\left(a+b+\frac{p}{1-p}+\frac{q}{1-q}\right) \eta^{(i)} \geqslant t\right) \rightarrow \exp \{-t\}
$$

npu $\mathrm{E} \xi_{1} \rightarrow 0$

ДокАЗАТЕЛьство. В нашем случае корни уравнения $\mathrm{E} \mu^{\xi_{1}}=1$ равны

$$
h_{1,2}=\frac{-L \pm \sqrt{L^{2}-4 C K}}{2 C}
$$

Здесь $L=L(1), C=C(1), K=K(1)$. Заметим, что $L^{2}-4 C K=(L+2 C)^{2}$. Обозначим $m=\mathrm{E} \xi_{1}$. Прямыми вычислениями устанавливаем:

$$
L+2 C=-(1-p)(1-q) m \text {. }
$$

Пусть для определенности $m>0$, тогда $L+2 C<0, h_{2}=1, h_{1}=-1-L / C$ и

$$
\theta=1-h_{1}=\frac{2 C+L}{C}=\frac{(1-p)(1-q) m}{p(1-r)+\alpha}
$$

Заметим, что коэффициент при $m$ в этом выражении допускает оценку

$$
\begin{aligned}
\frac{(1-p)(1-q)}{p(1-r)+\alpha} & =\frac{(1-p)(1-q)}{p(1-r)+\frac{(1-p)^{2}(1-r)}{2-p-q}+\frac{m(1-p)^{2}(1-q)}{2-p-q}} \\
& \leqslant \frac{(1-p)(1-q)}{p(1-r)+\frac{(1-p)^{2}(1-r)}{2-p-q}}=\frac{(1-p)(1-q)(2-p-q)}{(1-r)(1-p q)} \leqslant \frac{2}{1-r}
\end{aligned}
$$

Здесь использовалось выражение для $\alpha$, которое получается из системы уравнений

$$
\begin{gathered}
\frac{\alpha}{1-p}+\frac{\beta}{1-q}=1-r, \\
\frac{\alpha}{(1-p)^{2}}-\frac{\beta}{(1-q)^{2}}=m .
\end{gathered}
$$


Следовательно, при $1-r \geqslant \varepsilon>0$ коэффициент при $m$ ограничен и $\theta \rightarrow 0$ при $m \rightarrow 0$. Далее,

$$
d=(1-\theta)^{a+b-1}\left(1-\frac{\theta}{1-q}\right)\left(1-\frac{p \theta}{1-p+p \theta}\right),
$$

при $\theta \rightarrow 0$

$$
\begin{aligned}
\ln d & =-\theta(a+b-1)-\frac{\theta}{1-q}-\frac{\theta p}{1-p+p \theta}+o(\theta) \\
& =-\theta\left(a+b+\frac{p}{1-p}+\frac{q}{1-q}\right)+o(\theta) .
\end{aligned}
$$

Пусть $\theta_{1}=\theta\left(a+b+\frac{p}{1-p}+\frac{q}{1-q}\right)$. Для любого $t \geqslant 0$ найдется $k \in \mathbb{Z}_{+}$такое, что

$$
k \leqslant \frac{t}{\theta_{1}} \leqslant k+1
$$

Тогда

$$
\mathrm{P}\left(\theta_{1} \eta^{(1)} \geqslant t\right)=\mathrm{P}\left(\eta^{(1)} \geqslant \frac{t}{\theta_{1}}\right) \leqslant \mathrm{P}\left(\eta^{(1)} \geqslant k\right)=c_{1} d^{k-1}
$$

Заметим, что

$$
\begin{gathered}
c_{1}=\mathrm{P}\left(\eta^{(1)} \geqslant 1\right) \rightarrow 1, \\
d^{k-1} \rightarrow \exp \{-t\}
\end{gathered}
$$

при $m \rightarrow 0$. С другой стороны,

$$
\mathrm{P}\left(\theta_{1} \eta^{(1)} \geqslant t\right) \geqslant \mathrm{P}\left(\eta^{(1)} \geqslant k+1\right)=c_{1} d^{k} .
$$

Повторяя вычисления, получаем

$$
\lim _{m \rightarrow 0} c_{1} d^{k}=\exp \{-t\} .
$$

Доказательство для случая $i=1$ закончено. Случай $i=2$ рассматривается аналогично.

TeOPema 2. Пусть $\mathrm{P}\left(\xi_{1}=1\right)=\alpha, \mathrm{P}\left(\xi_{1}=-1\right)=\beta=1-\alpha$, тогда при $k \geqslant 1$

$$
\begin{aligned}
& \mathrm{P}\left(\eta_{n}^{(1)} \geqslant k\right)=\sum_{i=\gamma}^{\left[\frac{n+\gamma}{2}\right]} \frac{(2 \alpha)^{b}}{(4 \alpha \beta)^{k(a+b)}} 2^{\gamma}(\alpha \beta)^{i}\left\{\left(\begin{array}{c}
2 i-\gamma-1 \\
i-1
\end{array}\right)-\left(\begin{array}{c}
2 i-\gamma-1 \\
i
\end{array}\right)\right\}, \\
& \mathrm{P}\left(\eta_{n}^{(2)} \geqslant k\right)=\sum_{i=\widetilde{\gamma}}^{\left[\frac{n+\widetilde{\gamma}}{2}\right]} \frac{(2 \beta)^{a}}{(4 \alpha \beta)^{k(a+b)}} 2^{\widetilde{\gamma}}(\alpha \beta)^{i}\left\{\left(\begin{array}{c}
2 i-\widetilde{\gamma}-1 \\
i-1
\end{array}\right)-\left(\begin{array}{c}
2 i-\widetilde{\gamma}-1 \\
i
\end{array}\right)\right\},
\end{aligned}
$$

где числа а и $b$ челье, $-a \leqslant 0 \leqslant b, a+b>0, \gamma=2 k(a+b)-b, \widetilde{\gamma}=2 k(a+b)-a$, квадратные скобки означают челую часть числа. 
ДокАЗАТЕльство. Заметим, что при $\lambda=0$ правая часть (2) представляет собой выражение для производящей функции случайной величины $\tau_{k}^{+}$. Это дает возможность получить значения вероятностей событий $\left\{\tau_{k}^{+}=j\right\}$ и, в конечном итоге, прийти к соотношению (7), ибо имеет место очевидное равенство

$$
\mathrm{P}\left(\eta_{n}^{(1)} \geqslant k\right)=\sum_{j=1}^{n} \mathrm{P}\left(\tau_{k}^{+}=j\right) .
$$

Поэтому наша ближайшая цель состоит в разложении композиции операторов $\left(\left(J_{b}^{+} J_{-a}^{-}\right)^{k} e\right)(z, 1)$ в ряд по степеням $z$. Для выполнения условий теоремњ 2 положим $p=q=r=0$ в (3), (4). Тогда

$$
\left(\left(J_{b}^{+} J_{-a}^{-}\right)^{k} e\right)(z, 1)=\left(\frac{h_{1}(z)}{h_{2}(z)}\right)^{k(a+b)} \frac{1}{h_{1}^{b}(z)},
$$

где $h_{1,2}(z)=\frac{1 \pm \sqrt{1-4 \alpha \beta z^{2}}}{2 z \alpha}$. Далее находим

$$
\begin{aligned}
\left(\left(J_{b}^{+} J_{-a}^{-}\right)^{k} e\right)(z, 1) & =\left(\frac{1-\sqrt{1-4 \alpha \beta z^{2}}}{1+\sqrt{1-4 \alpha \beta z^{2}}}\right)^{k(a+b)} \frac{(2 z \alpha)^{b}}{\left(1-\sqrt{1-4 \alpha \beta z^{2}}\right)^{b}} \\
& =\frac{\left(1-\sqrt{1-4 \alpha \beta z^{2}}\right)^{2 k(a+b)-b}}{\left(4 \alpha \beta z^{2}\right)^{k(a+b)}}(2 z \alpha)^{b} \\
& =\left(1-\sqrt{1-4 \alpha \beta z^{2}}\right)^{2 k(a+b)-b} z^{b-2 k(a+b)} \frac{(2 \alpha)^{b}}{(4 \alpha \beta)^{k(a+b)}} .
\end{aligned}
$$

Пусть $\gamma=2 k(a+b)-b$. Тогда

$$
\begin{aligned}
(1- & \left.\sqrt{1-4 \alpha \beta z^{2}}\right)^{\gamma}=\sum_{j=0}^{\gamma}\left(\begin{array}{c}
\gamma \\
j
\end{array}\right)(-1)^{j}\left(1-4 \alpha \beta z^{2}\right)^{j / 2} \\
& =\sum_{j=0}^{\gamma}\left(\begin{array}{c}
\gamma \\
j
\end{array}\right)(-1)^{j} \sum_{i=0}^{\infty}(-1)^{i}\left(\begin{array}{c}
j / 2 \\
i
\end{array}\right)\left(4 \alpha \beta z^{2}\right)^{i} \\
& =\sum_{i=0}^{\infty}\left\{(-1)^{i}\left(4 \alpha \beta z^{2}\right)^{i}\left[\sum_{j=0}^{\gamma}\left(\begin{array}{c}
\gamma \\
j
\end{array}\right)(-1)^{j}\left(\begin{array}{c}
j / 2 \\
i
\end{array}\right)\right]\right\} \equiv \sum_{i=0}^{\infty}(-1)^{i}\left(4 \alpha \beta z^{2}\right)^{i} f_{i},
\end{aligned}
$$

где $f_{i}=\sum_{j=0}^{\gamma}\left(\begin{array}{l}\gamma \\ j\end{array}\right)(-1)^{j}\left(\begin{array}{c}j / 2 \\ i\end{array}\right)$. Напомним, что

$$
\left(\begin{array}{c}
j / 2 \\
i
\end{array}\right)=\frac{(j / 2-i+1)(j / 2-i+2) \cdots(j / 2)}{i !},
$$

если $i \leqslant j / 2$, и по определению эта величина равна нулю, если $i>j / 2$.

Из [7; формула 4.2.5.54] имеем

$$
f_{i}=\left\{\begin{array}{l}
(-1)^{i} 2^{\gamma-2 i}\left\{\left(\begin{array}{c}
2 i-\gamma-1 \\
i-1
\end{array}\right)\left(\begin{array}{c}
2 i-\gamma-1 \\
i
\end{array}\right)\right\}, \text { если } i \geqslant \gamma \\
\text { если } i<\gamma .
\end{array}\right.
$$


Таким образом,

$\left(\left(J_{b}^{+} J_{-a}^{-}\right)^{k} e\right)(z, 1)=\frac{(2 \alpha)^{b} 2^{\gamma}}{(4 \alpha \beta)^{k(a+b)}} \sum_{i=\gamma}^{\infty}(\alpha \beta)^{i} z^{2 i-\gamma}\left\{\left(\begin{array}{c}2 i-\gamma-1 \\ i-1\end{array}\right)-\left(\begin{array}{c}2 i-\gamma-1 \\ i\end{array}\right)\right\}$.

Вычисления, аналогичные приведенным вьше, дают

$\left(\left(J_{-a}^{-} J_{b}^{+}\right)^{k} e\right)(z, 1)=\frac{(2 \alpha)^{a} 2^{\tilde{\gamma}}}{(4 \alpha \beta)^{k(a+b)}} \sum_{i=\widetilde{\gamma}}^{\infty}(\alpha \beta)^{i} z^{2 i-\widetilde{\gamma}}\left\{\left(\begin{array}{c}2 i-\widetilde{\gamma}-1 \\ i-1\end{array}\right)-\left(\begin{array}{c}2 i-\widetilde{\gamma}-1 \\ i\end{array}\right)\right\}$,

здесь $\widetilde{\gamma}=2 k(a+b)-a$ и, следовательно, в условиях теоремы 2

$$
\begin{aligned}
& \mathrm{P}\left(\eta_{n}^{(1)} \geqslant k\right)=\sum_{i=\gamma}^{\left[\frac{n+\gamma}{2}\right]} \frac{(2 \alpha)^{b}}{(4 \alpha \beta)^{k(a+b)}} 2^{\gamma}(\alpha \beta)^{i}\left\{\left(\begin{array}{c}
2 i-\gamma-1 \\
i-1
\end{array}\right)-\left(\begin{array}{c}
2 i-\gamma-1 \\
i
\end{array}\right)\right\}, \\
& \mathrm{P}\left(\eta_{n}^{(2)} \geqslant k\right)=\sum_{i=\widetilde{\gamma}}^{\left[\frac{n+\tilde{\gamma}}{2}\right]} \frac{(2 \beta)^{a}}{(4 \alpha \beta)^{k(a+b)}} 2^{\widetilde{\gamma}}(\alpha \beta)^{i}\left\{\left(\begin{array}{c}
2 i-\widetilde{\gamma}-1 \\
i-1
\end{array}\right)-\left(\begin{array}{c}
2 i-\widetilde{\gamma}-1 \\
i
\end{array}\right)\right\},
\end{aligned}
$$

что и требовалось доказать.

Далее будем изучать предельное поведение распределения числа пересечений полосы для случайных блужданий общего вида с нулевым сносом.

Пусть $C[0,1]$ - пространство непрерьвных функций на $[0,1]$ с метрикой $\rho=$ $\sup _{t}|x(t)-y(t)|$. Для $x \in C[0,1]$ обозначим через $f_{A, B}^{(1)}(x), f_{A, B}^{(2)}(x)$ число пересечений полосы $-A \leqslant y \leqslant B,-A \leqslant 0 \leqslant B$, траекториями $(t, x(t))$ на координатной плоскости $(t, y)$ соответственно снизу вверх и сверху вниз. Определим случайные величины $\chi_{A, B}^{(1)}=f_{A, B}^{(1)}(W), \chi_{A, B}^{(2)}=f_{A, B}^{(2)}(W)$, где $W(t)$ - стандартный винеровский процесс на $[0,1]$.

ТЕОРема 3. Пусть $\xi_{1}, \xi_{2}, \ldots$ - независимые одинаково распределенные случайнье величины такие, что $\mathrm{E} \xi_{1}=0, \mathrm{E} \xi_{1}^{2}=1$, и пусть $a=A \sqrt{n}, b=B \sqrt{n}$, $c=C \sqrt{n}, d=D \sqrt{n}, C \leqslant D$. Тогда для любого $k \geqslant 1$ при $n \rightarrow \infty$

$$
\mathrm{P}\left(\eta_{n}^{(i)} \geqslant k, S_{n} \in[c, d]\right) \rightarrow \mathrm{P}\left(\chi_{A, B}^{(i)} \geqslant k, W(1) \in[C, D]\right),
$$

при этом

$$
\begin{aligned}
& \mathrm{P}\left(\chi_{A, B}^{(1)} \geqslant k, W(1) \in[C, D]\right) \\
& \quad=\Phi(N \vee L)-\Phi(M \vee L)+\Phi((2 L-M) \vee L)-\Phi((2 L-N) \vee L), \\
& \mathrm{P}\left(\chi_{A, B}^{(2)} \geqslant k, W(1) \in[C, D]\right) \\
& \quad=\Phi\left(N \vee L_{1}\right)-\Phi\left(M \vee L_{1}\right)+\Phi\left(\left(2 L_{1}-M\right) \vee L_{1}\right)-\Phi\left(\left(2 L_{1}-N\right) \vee L_{1}\right),
\end{aligned}
$$

гдe

$$
\Phi(x)=\frac{1}{\sqrt{2 \pi}} \int_{-\infty}^{x} \exp \left\{-\frac{t^{2}}{2}\right\} d t
$$

$L=2 k(A+B)-B, L_{1}=2 k(A+B)-A, M=C+2 k(A+B), N=D+2 k(A+B)$, $M \vee N=\max (M, N), i=1,2$. 
ДокАЗАтЕльство. Установим первое соотношение. Покажем, что отображение, переводящее точку $x$ из $C[0,1]$ в точку $\left(f_{A, B}^{(i)}(x), x(1)\right)$ пространства $\mathbb{R}^{2}$ непрерывно всюду, за исключением множества винеровской меры 0. Для этого достаточно доказать данное утверждение для функции $f_{A, B}^{(i)}, i=1,2$. Пусть $x$ - точка разрыва функции $f_{A, B}^{(i)}$, тогда траектория $x(t)$ должна касаться верхней границы полосы снизу или нижней границы сверху при некотором $t$. Непрерывность всюду, кроме множества винеровской меры 0 , следует теперь из непрерывности функции распределения верхней и нижней грани $W(t)$ на $(t-\varepsilon, t+\varepsilon)$ для любого $t$ и $\varepsilon>0$. Действительно, пусть $v$ - винеровская мера, и пусть событие $A_{1}$ означает, что траектория $W(t)$ в некоторой точке касается уровня $-A$ сверху, а событие $B_{1}$ состоит в том, что $W(t)$ касается уровня $B$ снизу в некоторой точке. Тогда

$$
v\left(x(t): x(t) \text { есть точка разрыва } f_{A, B}^{(i)}\right) \leqslant \mathrm{P}\left(A_{1}\right)+\mathrm{P}\left(B_{1}\right) .
$$

Далее,

$$
\begin{aligned}
\mathrm{P}\left(B_{1}\right) & =\mathrm{P}\left(\bigcup_{n=1}^{\infty} \bigcup_{k=1}^{n}\left\{\sup _{t \in\left[\frac{k-1}{n}, \frac{k}{n}\right]} W(t)=B\right\}\right) \\
& \leqslant \sum_{n=1}^{\infty} \mathrm{P}\left(\bigcup_{k=1}^{n}\left\{\sup _{t \in\left[\frac{k-1}{n}, \frac{k}{n}\right]} W(t)=B\right\}\right)=0 .
\end{aligned}
$$

Аналогично получаем $\mathrm{P}\left(A_{1}\right)=0$. Пусть для $n \geqslant 1$ случайная функция $x_{n}(t)$ построена по отрезкам случайного блуждания $\left\{\left(m, S_{m}\right)\right\}_{m=0}^{n}$ следующим образом: полагаем $x_{n}(j / n)=(1 / \sqrt{n}) S_{j}$ для точек вида $j / n, j=0,1, \ldots, n$. Для прочих точек $t \in[0,1]$ определим $x_{n}(t)$ с помощью линейной интерполяции: если $t \in[(i-1) / n, i / n]$, то

$$
x_{n}(t)=\frac{1}{\sqrt{n}} S_{i-1}+n\left(t-\frac{i-1}{n}\right) \frac{1}{\sqrt{n}} \xi_{i} .
$$

Таким образом, первое утверждение теоремы следует из слабой сходимости $x_{n}$ к $W$ и непрерьвности $f_{A, B}^{(i)}$ почти всюду. Найдем явный вид совместного распределения случайных величин $\chi_{A, B}^{(2)}$ и $W(1)$. Для этого установим равенства

$$
\begin{aligned}
& \mathrm{P}\left(\chi_{A, B}^{(2)} \geqslant k, W(1) \in[C, D]\right) \\
& =\mathrm{P}\left(\sup _{t \in[0,1]} W_{2 k}(t) \geqslant 2 k(A+B)-A,\right. \\
& \left.\quad W_{2 k}(1) \in[C+2 k(A+B), D+2 k(A+B)]\right) \\
& =\mathrm{P}\left(\sup _{t \in[0,1]} W(t) \geqslant 2 k(A+B)-A, W(1) \in[C+2 k(A+B), D+2 k(A+B)]\right) .
\end{aligned}
$$


Здесь для любого $k \geqslant 1$ процесс $W_{2 k}(t)$ построен следующим образом. Для процесса $W(t)$ пусть $\tau_{1}^{B}$ - момент первого пересечения уровня $B, \tau_{1}^{-A}$ - первый после $\tau_{1}^{B}$ момент пересечения уровня $-A, \tau_{2}^{B}$ - первый после $\tau_{1}^{-A}$ момент пересечения уровня $B$ и так далее до $\tau_{k}^{-A}$. Положим $W_{1}(t)=W(t)$ при $t<\tau_{1}^{B}$, для $t \geqslant \tau_{1}^{B}$ процесс $W_{1}(t)$ является зеркальным отражением процесса $W(t)$ относительно уровня $B$, т.е. $W_{1}(t)=2 B-W(t)$ при $t \geqslant \tau_{1}^{B}$. Процесс $W_{2}(t)$ совпадает с $W_{1}(t)$ при $t<\tau_{1}^{-A}$, а при $t \geqslant \tau_{1}^{-A}$ процесс $W_{2}(t)$ является зеркальньм отражением процесса $W_{1}(t)$ относительно уровня $B+(A+B)$, т.е. $W_{2}(t)=2(B+$ $(A+B))-W_{1}(t)$ при $t \geqslant \tau_{1}^{-A}$. Заметим, что процесс $W(t)$ пересекает хотя бы один раз рассматриваемую полосу сверху вниз тогда и только тогда, когда процесс $W_{2}(t)$ достигает уровня $B+(A+B)$. Продолжая далее эти построения, получаем на $k$-м шаге процесс $W_{2 k}(t)=W_{2 k-1}(t)$ при $t<\tau_{k}^{-A}$ и $W_{2 k}(t)=2(B+$ $(2 k-1)(A+B))-W_{2 k-1}(t)$ при $t \geqslant \tau_{k}^{-A}$. События $\left\{\chi_{A, B}^{(2)} \geqslant k, W(1) \in[C, D]\right\}$ и $\left\{\sup _{t \in[0,1]} W_{2 k}(t) \geqslant 2 k(A+B)-A, W_{2 k}(1) \in[C+2 k(A+B), D+2 k(A+B)]\right\}$ совпадают в силу наличия взаимно однозначного соответствия между траекториями процессов $W(t)$ и $W_{2 k}(t)$. Второе равенство в $(9)$ справедливо в силу того, что процессы $W(t)$ и $W_{2 k}(t)$ одинаково распределены (см. [8; гл. 6, §3]). Вероятность события $\left\{\sup _{t \in[0,1]} W(t) \geqslant 2 k(A+B)-A, W(1) \in[C+2 k(A+B), D+2 k(A+B)]\right\}$ хорошо известна, она приведена в формулировке теоремы. Аналогично находим совместное распределение случайных величин $\chi_{A, B}^{(1)}$ и $W(1)$. Теорема 3 доказана.

Заметим попутно, что из доказанной теоремы легко следует известный результат о том, что число пересечений уровня траекторией винеровского процесса бесконечно. Действительно, пусть $A=B=1 / k$, тогда для любого $k \geqslant 1$

$\mathrm{P}\left(\chi_{0,0}^{(i)} \geqslant k\right) \geqslant \mathrm{P}\left(\chi_{A, B}^{(i)} \geqslant k\right)=\mathrm{P}\left(\sup _{t \in[0,1]} W(t) \geqslant 4-\frac{1}{k}\right) \geqslant \mathrm{P}\left(\sup _{t \in[0,1]} W(t) \geqslant 4\right)>0$.

\section{Список литературы}

1. Лотов В.И. Об одном подходе в двуграничных задачах // Статистика и управление случайными процессами. М.: Наука, 1989. С. 117-121.

2. Гіхман Й. I. Асимптотичні розподіли числа перетинів випадковою функціею границі даноі області // Вісн. Киів. ун-ту. Сер. астрон., матем., механ. 1958. Т. 1. № 1. С. 25-46.

3. Скороход A.В., Слободенюк Н.Г. Предельные теоремы для случайных блужданий. Киев: Наукова думка, 1970.

4. Chung K. L., Hunt G. A. On the zeros of $\sum_{1}^{n} \pm 1 / /$ Ann. of Math. (2). 1949. V. 50. № 2. P. $385-400$.

5. Боровков А. А. Теория вероятностей. М.: Наука, 1986.

6. Лотов В.И. Асимптотический анализ распределений в двуграничных задачах. I // Теория вероятностей и ее прим. 1979. Т. 24. № 3. С. 475-485.

7. Прудников А. П., Брычков Ю. А., Маричев О. И. Интегралы и ряды. М.: Наука, 1981.

8. Гихман И.И., Скороход А.В. Введение в теорию случайных процессов. М.: Наука, 1977.

Институт математики им. С. Л. Соболева СО РАН,

Поступила в редакцию Новосибирск 19.03 .2002 и 21.03 .2003

E-mail: lotov@math.nsc.ru 